\title{
A national strategic plan for reducing the burden of sexually transmitted infections in Israel by the year 2025
}

Daniel Chemtob ${ }^{1 *}$ (D), Dan Gandacu2 2 Zohar Mor ${ }^{3}$, Itamar Grotto ${ }^{4}$, Emilia Anis ${ }^{2}$ and Elliot Rosenberg ${ }^{5}$

\begin{abstract}
Background: There is on ongoing debate in the literature regarding the real burden of STIs (sexually transmitted infections) in Western countries and the proper strategies needed to estimate and to prevent them. Our purpose is to present an evidence-based national strategic plan for STI prevention in Israel through assessing the current burden of illness, leading international preventive strategies, and practical policymaking experience.
\end{abstract}

Methods: Epidemiologic and health policy data on STIs were analyzed from various sources: a) systematic national surveillance data for the years 2002-2014; b) the international scientific literature (published between 2002-16; keywords: Sexually Transmitted Diseases (STD) (or STI) AND prevention AND intervention AND gonorrhea OR chlamydia OR syphilis; c) internal Ministry of Health $(\mathrm{MOH})$ analyses and reports, and d) expert opinion.

Results: Incidence rates in Israel of Chlamydia trachomatis (chlamydia), Neisseria gonorrhea (gonorrhea) and Treponema pallidum (syphilis) are lower than in most Western countries. However, rates vary among population subgroups: chlamydia, gonorrhea and syphilis are higher in Jews than in non-Jews, and this gap has increased for chlamydia over the past decade. Primary and secondary syphilis rates have increased among men having sex with men (MSM). It is likely that STIs are under-reported and that incidence is even rising due to migration.

A key recommendation is the establishment of an active surveillance system of STIs, utilizing active case finding in high risk populations, along with regular contact with STI clinics run by the four national health management organizations and by the $\mathrm{MoH}$. As with most European countries, the low prevalence of chlamydia and gonorrhea does not justify population-wide screening. Conversely, the increasing incidence of syphilis among MSM should lead to regular screening among this group.

Conclusions: A national STIs prevention strategy for the year 2025 was presented. Although the current burden of illness is low relatively to other Western countries, this is thought to reflect a certain degree of underreporting. These and other gaps suggest a need for focused epidemiologic and health services research to better characterize health risk behaviors as well as provider practice patterns. Innovative implementation strategies have been described, together with the capacity building components needed for developing specific and implementable policy recommendations for the year 2025.

\footnotetext{
* Correspondence: daniel.chemtob@moh.health.gov.il;

daniel.chemtob@mail.huji.ac.il

${ }^{1}$ Department of Tuberculosis and AIDS, Ministry of Health, Jerusalem, Israel

Full list of author information is available at the end of the article
} 


\section{Introduction}

The incidence rates of the three main sexually transmitted infections (STIs) (other than HIV) in Israel- Chlamydia trachomatis (chlamydia), Neisseria gonorrhea (gonorrhea), and Treponema pallidum (syphilis) are lower than those in most western countries [1, 2]. However, these rates may actually be higher due to under-reporting of new cases. Additionally, if appropriate preventive and therapeutic measures are not implemented in a timely fashion, rates may rise due to the following factors: (1) importation of additional cases by migrants from countries with either high prevalence levels of STIs or from countries experiencing recent epidemic outbreaks followed by dissemination within the veteran population; (2) international travel by a native and migrant residents; (3) a significant prevalence of commercial sex work (CSW) in urban areas [2].

Meeting these challenges requires a thorough mapping of the STI epidemiology in Israel, the development of a comprehensive preventive strategy, as well as a practicable implementation program. These challenges have been addressed within the context of a national prevention initiative entitled 'Healthy Israel 2020', which aims to increase the life expectancy and the quality of life of Israel's citizens, while reducing health inequity (specific recommendations for STIs have been postponed to 2025). Topic areas for the entire initiative were selected on the basis of their respective burdens of disease [3]. One of the topic areas covered the prevention of infectious diseases; a subcommittee thereof focused on the prevention of Tuberculosis (TB), HIV, and STIs. Recommendations on the topics of TB-HIV have been published elsewhere [4]. The current integrative article focuses on the development of national targets and objectives for the reduction of the disease burden due to STIs by the year 2025, as well as an evidence-based, and implementable strategic plan to achieve that aim.

\section{Methods}

Data on the epidemiology, preventive interventions and best practice implementation strategies for the prevention of the three main STIs in Israel were analyzed and accessed from various sources. Systematic surveillance data collected by the Division of Epidemiology in the Israeli Ministry of Health $(\mathrm{MoH})$ for the years 20022014 was used to describe epidemiological STI data on the Israeli population in addition to internal $\mathrm{MOH}$ analyses and reports ("grey literature"). Country-level STI incidence rates were collected from the databases of the World Health Organization (WHO) Regional Office for Europe, the US CDC, and the Australian National Notifiable Disease Surveillance System. Primary and secondary preventive interventions to reduce the burden of STIs published between 2002 and 2016 were accessed from the English language scientific literature by entering the following keywords into PubMed: Sexually Transmitted Diseases (STD) (or STI) AND prevention AND intervention AND gonorrhea OR chlamydia OR syphilis. Twenty one articles describing preventive interventions were retrieved, primarily from European countries and the United States (US) for each specified STI (gonorrhea, chlamydia or syphilis). Particular attention was focused on data/recommendations issued by evidence-based international and other national preventive health oriented organizations such as, the WHO, the Cochrane collaboration, the US Preventive Services Task Force, the Task Force for Community Preventive Services, as well as various European countries.

Throughout our investigation, numerous discussions were held between the $\mathrm{MoH}$ staff, several stakeholders (both governmental and non-governmental), and senior medical professionals; these were further elaborated upon by the Healthy Israel 2020 sub-committee on STIs in order to develop a coherent, evidence-based national plan to reduce the burden of STIs in Israel.

\section{Results \\ Epidemiology \\ International}

Incidence rates for the three main STIs vary widely between countries and show different trends over time. When analyzing the 53 WHO European countries, many did not report STI incidences annually to the WHO European region [1]. Incidence trends (per 100,000 population) from countries who regularly reported to WHO European region, including Israel, were accessed for the years 2006-2013 [1]. In addition, trends in the US and Australia were also analyzed for this period $[5,6]$.

Chlamydia Incidence rates (per 100,000 population) in the $27 \mathrm{WHO}$ European region countries which reported data for the year 2012 were highest in the Scandinavian countries; Iceland led this region with 576 cases/ 100,000 . Rates tended to decrease merely from north to south: the United Kingdom - 378, Belgium - 43, Israel 10.8, and Spain a mere 1.9. The high rates of Chlamydia and discrepancies between Scandinavian countries and other countries are also a partial reflection of ascertainment and reporting biases.

Most of the WHO European region European countries reviewed showed a distinct increase over the years 20062013. This increase was noted in other countries as well: the rate in the USA increased from 344/100,000 in 2006 to $443 / 100,000$ in 2013 [5]; and in Australia, increased from $230 / 100,000$ in 2006 to $363 / 100,000$ in 2013 [6].

Gonorrhea Incidence rates (per 100,000 population) in the $34 \mathrm{WHO}$ European region countries which reported data for the year 2012 were much lower than those of chlamydia, ranging from 45.5 in the United Kingdom 
and 36.5 in the Russian Federation, to 0.45 in Italy, 0.16 in Montenegro and 0.11 in Bosnia [1]. Many countries experienced fluctuations during the years 2006-2013. Israel's rates fluctuated between 2.1 and 4.1/100,000 during this period. US rates decreased from 2006 to 2009 , but then began to rise again. US rates were significantly higher than those in the European region, reaching 105.3/100,000 in 2013 [5]. Australia has also experienced fluctuating rates, with $41.6 / 100,000$ in $2006,35.7 / 100,000$ in 2008 , but rising to $64.5 / 100,000$ in 2013 [6].

Primary and secondary early syphilis Incidence rates (per 100,000 population) in the 25 WHO European region countries which reported data for the year 2012 were all of the same order of magnitude, ranging from 11.9 in Georgia to 1.3 in Sweden [1] (except the Republic of Moldova which reported an exceptionally high rate of 64.7). Israel, though, reported an even lower rate: 0.9 / 100,000 . Since 2006, slightly more countries have noted an increase rather than a decrease in incidence trends, but in most they fluctuated. The US rate of 5.5/100,000 in 2013 is higher than that seen in almost all European countries [5]. Australia has experienced fluctuating rates, with 4.3 in 2006 rising to 6.8 in 2007, dropping to 5.1 in 2010, but then rising again to 7.6 in 2013 [6].

On the basis of current passive surveillance, incidence rates of chlamydia, gonorrhea and syphilis are low in Israel relative to northern European countries [1], the USA [5] and Australia [6], and are roughly similar to those reported by southern Mediterranean European countries.

\section{Israel: an in-depth analysis}

In 1994, Chlamydia was added to the list of STIs already defined in Israel as reportable diseases. STI incidence rates by population groups (for the period 2002-2014) appear in Figs. 1, 2 and 3.

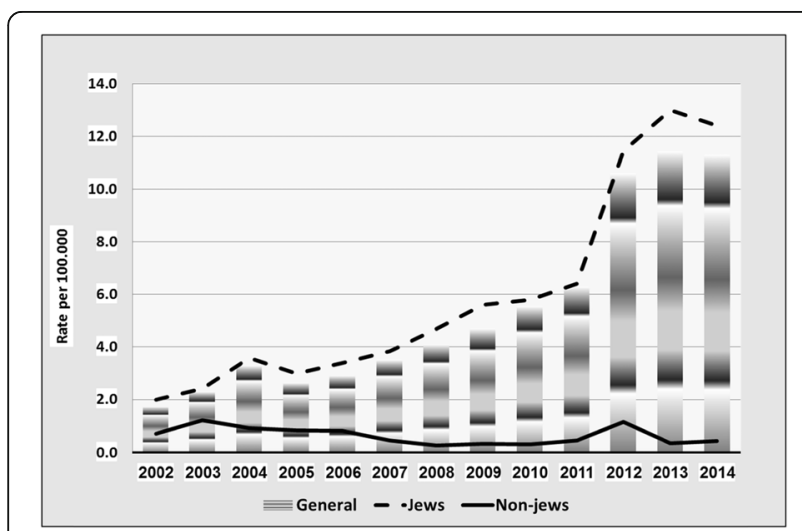

Fig. 1 Incidence of Chlamydia trachomatis infection, by year and by population groups, Israel 2002-2014

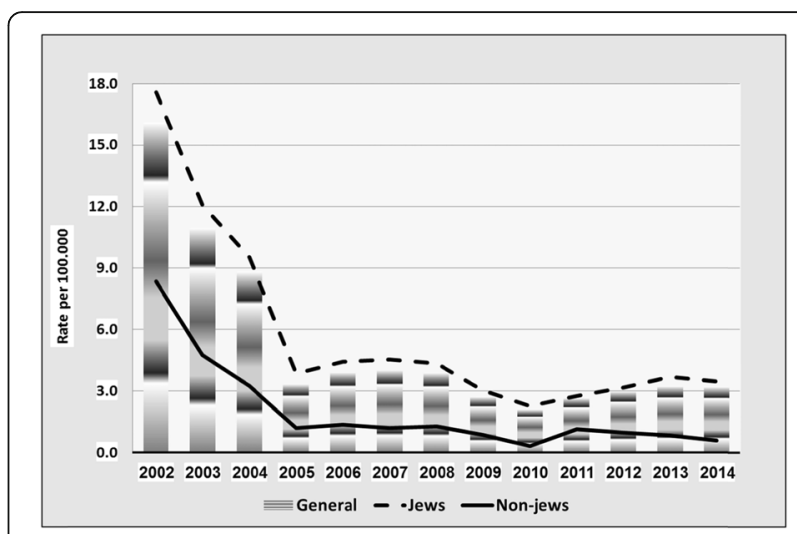

Fig. 2 Incidence of Neisseria gonorrhea by year and by population groups, Israel 2002-2014

The main findings were as follows:

- Rates of chlamydia are appreciably higher in Jews than in non-Jews. This difference has increased over the past decade. Overall, rates have markedly increased in recent years (from 2.9/100,000 in 2006 to $11.3 / 100,000$ in 2014).

- Rates of gonorrhea have decreased in the last decade, and are approximately three times higher in Jews than in non-Jews. Males account for the majority of the cases (male to female ratio $>4$ ). Increasing requests for bacteriologic testing of pharyngeal specimens, especially in men having sex with men (MSM) and in CSW, may indicate an increasing trend in the transmission of pharyngeal gonorrhea [7].

- The rates of syphilis are roughly three to four times higher in Jews than in non-Jews. Between the years 2002-2011, rates have dropped by over

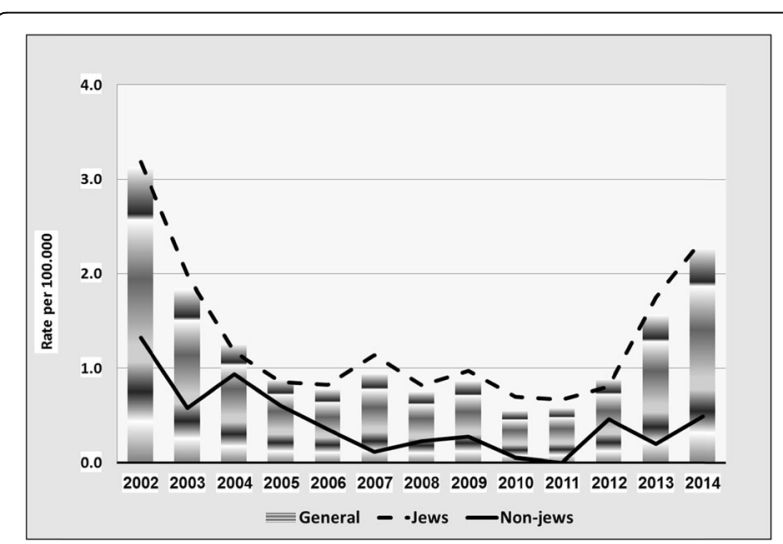

Fig. 3 Incidence of Treponema palladium Infections by year and by population groups, Israel 2002-2014 
$70 \%$ in the Jewish population and by $80 \%$ in the non-Jewish population, before increasing back since 2012 and forming a U-curve, especially prominent among the Jewish population. This recent increase is largely influenced by an increase of reported new male cases, which are assumed to be mostly among MSM, the group at the greatest risk of infection in recent years [8].

- The number of cases of syphilis among HIV-infected MSM increased from 0 to 2 cases/year in the 1990-2000 period to 10-18 cases/year during the 2005-2009 period. In the Tel Aviv district, where $90 \%$ of the patients were MSM, the number of males infected with syphilis increased from 5 cases in 2005 to 40 cases in 2009. In the $\mathrm{MoH}$ STI clinic in Tel-Aviv, 1,064 (22\%) MSM and 3,755 (78\%) heterosexuals were tested. Positivity rates for HIV, urethral $N$. gonorrhea and infectious syphilis in MSM were higher than in heterosexuals $(2.5 \%, 2.5 \%$. $0.7 \%$ vs. $1.6 \%, 1.3 \%$, $0.3 \%$, respectively), while urethral C. trachomatis was higher in heterosexuals than in MSM (2.7 vs. $1.4 \%$, respectively) [9].

- At National level, the peak age of incidence of all three STIs was 15-44, where the 25-34 year old age group suffered the greatest burden of infection. Further analysis of incidence trends in these three major STIs by age group (15-24; 25-34; 35-44) appears in a recent publication [2].

\section{Setting the $\mathbf{2 0 2 5}$ objectives and targets}

The aforementioned data sources resulted in the generation of the following baseline incidence rates for the three STIs of interest in 2014:

- Chlamydia: $11.3 / 100,000$

- Gonorrhea: 3.2/100,000

- Primary and secondary syphilis: $2.3 / 100,000$

The current Israeli STI surveillance system is predominantly passive and therefore the generated data is inherently incomplete due to partial reporting and capture of disease incidence data. Conversely, when setting target goals and national objectives to decrease the rates and/or the burden of disease, it is critical to establish more authoritative baseline values. These limitations led to the decision by the authors of this article to defer the definition of the 2025 objectives and target values until more accurate baseline values could be generated.

\section{Interventions \\ Primary prevention}

A. Evidence of effectiveness

1. Clinically-based

High intensity behavioral counseling interventions (contact time of over 2 hours) targeted to sexually active adolescents and adults at increased risk for STIs reduced the incidence of STI's when assessed 12 months post-counseling [10].

Note: Adults at increased risk included those with current STIs or infections within the past year, or those with multiple concurrent sexual partners, and adults who did not consistently use condoms.

2. Community-based

2.1 Comprehensive risk reduction (CRR) interventions delivered in schools or in community settings to groups of adolescents (aged 10-19) were effective when including one of the following approaches, as appropriate (and may include components such as condom distribution and STI testing):

- Propose several behaviors, including sexual risk reduction strategies, but suggest abstinence as the behavior of choice;

- Recommend both abstinence and sexual risk reduction as equivalent strategies;

- Advocate sexual risk reduction strategies as a sole or at least as a primary strategy [11].

2.2 Community-based youth development behavioral interventions coordinated with community service to reduce sexual risk behaviors in adolescents These interventions address a broad range of health and wellness issues. They may not necessarily include components that are focused directly on pregnancy and STI prevention. Key components include social, emotional, or cognitive competence training that promotes pro-social norms, improved decision making, self-determination, and communication skills. These interventions serve to strengthen positive bonding experiences between youth and their peers or non-parental role models.

The community service component is broad. Scheduling a variety of activities in community settings such as nursing homes, hospitals, and homeless shelters would be appropriate [12]. Community and school-based interventions are resource intensive. Before implementing 
such a program, cost-effectiveness analysis and small-scale piloting within communities would be necessary.

\section{B. International recommendations} WHO

The World Health Assembly endorsed the global strategy for the prevention and control of STIs in May 2006. The strategy urges all countries to control the transmission of STIs by implementing a number of interventions, including the following [13]:

- Involvement of all relevant stakeholders, including the private sector and the community, in prevention and care of STIs;

- Specific services for populations with frequent or unplanned high-risk sexual behaviors - such as Commercial Sex Workers (CSW), adolescents, military personnel, substance users and prisoners;

- Prevention by promoting safer sexual behaviors;

- General access to quality condoms at affordable US prices or free of charge for specific populations.

- The US Preventive Services Task Force (USPSTF) recommends high-intensity behavioral counseling to prevent STIs for all sexually active adolescents and for adults at increased risk for STIs. Grade: B Recommendation [10].

- The Community Preventive Services Task Force (CPSTF) recommends both CRR interventions delivered in school or in community settings to groups of adolescents [11], as well as youth development behavioral interventions coordinated with community service [14].

Israel

The Israeli Task Force on Health Promotion and Disease Prevention (a branch of the Scientific Council Israeli Medical Association) recommends for all 13-19 year olds to be counseled in matters regarding sexual behavior, STIs and contraceptive use. This is optional for 20-39 year-olds. No information is provided about the manner (methodology, training, frequency, duration etc.) in which this counseling is to be performed [15].

\section{Secondary prevention (screening)}

A. Evidence of efficacy

1. Chlamydia

Two randomized controlled trials demonstrated a reduction in medical complications in women members of a Health Maintenance Organization
(HMO) and in a high school that implemented Chlamydia screening $[16,17]$. A recent summary article from the US CDC justified screening sexually active young, as well as high risk older females, due to the large and costly burden of preventable illness (presenting as pelvic inflammatory disease (PID) and its sequela, tubal infertility), the asymptomatic nature of the infection in females, the ease of diagnosis with nucleic acid amplification tests, the highly efficacious treatment options, and the randomized trial data showing a reduction in PID incidence following screening [18]. Conversely, a systematic review did not find evidence for even opportunistic screening of women younger than age 25 , if not considered as high risk [19]. A Cochrane review found Chlamydia screening to have only a modest effect in reducing PID risks at the individual level, but had no effect on epididymitis or on infection levels among in men and women [20].

2. Gonorrhea Indirect evidence shows that screening sexually active women age 24 and younger, as well as older women at increased risk of infection (those with a new or more than one sexual partner, a sexual partner infected with an STI, inconsistent condom use, a history of previous or coexisting STIs, or those exchanging sex for money of drugs) may prevent other complications associated with gonococcal infection, such as pelvic inflammatory disease and its sequelae [21].

3. Syphilis

Screening tests for syphilis can adequately diagnose the disease. Effective and cheap antibiotic treatment is also available for cure. High risk individuals (including MSM, CSW, and adults in correctional facilities) have a higher pre-test probability of being diagnosed with syphilis. Despite the above, there are insufficient data to conclusively prove that screening reduces syphilis-related morbidity [22].

The recommendation to screen pregnant women is based upon observational evidence that screening decreases the proportion of newborns with clinical manifestations of syphilis infection and those with positive serology [23].

\section{B. Cost-Effectiveness}

In a review of 55 cost-effectiveness studies focused primarily on Chlamydia and HIV interventions, one-on-one interventions such as counseling (and screening) were proven to be cost-effective [24]. An editorial on the article [25] noted that these cost-effectiveness calculations were conservative 
because the benefits of STI prevention such as reduction of HIV incidence and productivity losses were excluded. A cost-utility analysis of Chlamydia screening calculated that the incremental costeffectiveness ratio relative to the next most effective strategy would cost less than $\$ 25,000$ USD/QALY for annual screening followed by semi-annual screening for those with a history of infection, thus classifying it as very cost effective [26]. This has been corroborated by a more recent United Kingdom (UK) calculation based on modeling: it was estimated that it would cost $£ 506$ (=US $\$ 776$ - Conversion rate as of April 15, 2013) per infection treated [27]. Israeli cost-effectiveness data is not yet available.

C. Screening policies in selected western countries US

Chlamydia and Gonorrhea The USPSTF recommends that all sexually active women aged 24 and younger, as well as for older women at high risk for STI be screening for chlamydia and gonorrhea. High risk is defined as those with one of the following risk factors: those with a history of previous or concurrent STI, those with a new or more than one sexual partner, a sex partner who is currently infected with STI, sporadic (inconsistent) use of condoms, and those who exchange sex for drugs or money.

Grade: B recommendation [21].

Syphilis

The USPSTF strongly recommends that persons at increased risk for syphilis infection be screened by clinicians. These include MSM engaged in high-risk sexual behavior, CSW, persons who exchange sex for drugs, and those in adult correctional facilities.

Grade: A Recommendation [28].

The USPSTF strongly recommends all pregnant women be screened for syphilis infection.

Grade: A Recommendation [29].

EU (European Union)

Chlamydia

In guidance presented in June 2009, The European Centre for Disease Prevention and Control (ECDC) recommended a step-by-step chlamydia control strategy whereby primary prevention, case management, and opportunistic testing to specified sub-populations attending clinical services be carried out, and evaluated, alongside the development of both patient management infrastructures, and quality controls before population-based screening be enacted [30].
Most European countries lack a national screening program for Chlamydia for asymptomatic individuals [30]. A national Chlamydia screening program was put into place in the UK in 2007 [31] for women under age 25 attending various clinical and non-clinical settings (e.g., universities and sporting events). A pilot program of annual postal invitation was introduced in three regions of the Netherlands in 16-29 year-olds in early 2008 [30]. A registerbased screening program using mailed homecollected specimens is planned in Norway [30]. Opportunistic screening is widespread in Sweden but it lacks national coordination and is conducted on a county basis. Several northern European countries perform opportunistic testing of asymptomatic individuals, e.g., Denmark tests people with frequent sex partner change and women aged 25 and under before an IUD insertion (although in two of sixteen communities in the country proactive screening has been introduced - via postal invitation) [30]. Iceland screens all women presenting for termination of pregnancy and egg or sperm donors [30]. Canada recommends populationwide screening [32]. Since 2005, Australia has been moving in this direction [33].

Gonorrhea

UK (United Kingdom) [34]

There is no basis to support widespread, unselected screening for gonorrhea where only meager evidence for selective community screening exists in the UK. STI data is limited for those treated outside the genitourinary medical (GUM) clinics and prevalence studies are rare. The prevalence of gonorrhea infection varies widely between and within communities and patient populations. Gonorrhea diagnoses and subsequent complications are infrequent compared to chlamydia. Higher prevalence of infection than the general population is found among inner-city residents, STIs clinics attendees, military personnel, prisoners and MSM. The immediate health benefits from an accurate gonorrhea diagnosis are the subsequent reduction of HIV transmission or acquisition risk, support such an intervention. However, the health benefits must be weighed in light of the cost and adverse effects of screening. Localized interventions targeted at the core high-risk groups are likely to be more costeffective and beneficial than unselected community-wide screening. 
Syphilis [35]

Routine tests for syphilis should be taken in all pregnant women, those donating blood, and in the following high risk groups for syphilis infection: (a) all patients who are newly diagnosed with a STI; (b) persons infected with HIV;(c) patients with hepatitis B or C; (d) patients suspected of early neurosyphilis (i.e., unexplained sudden visual loss [uveitis], unexplained sudden deafness [otitis] or meningitis); (e) patients who engage in sexual behavior that puts them at risk (e.g. MSM, CSW, and all those individuals at higher risk of acquiring STIs). Israel

1. Israeli Task Force for Health Prevention and Disease Prevention [15]:

All pregnant women should be tested for syphilis (with the VDRL - Venereal Disease Research Laboratory - test or by ELISA enzyme-linked immunosorbent assay). Serologic testing should be considered for pregnant women that are high-risk for Chlamydia and no recommendation was issued regarding screening women for gonorrhea.

2. Israeli Ministry of Health

Chlamydia and gonorrhea

Due to the relatively low incidence of chlamydia and gonorrhea in Israel, a population-wide screening program is unjustified. Continued prevalence surveys for specific high-risk populations and various population substrata will be used to guide future policy decisions.

Evidence-based routine surveillance and case management should continue.

Syphilis

Screening is recommended for the following high risk populations:

a. Pregnant women

b. MSM

c. Persons who exchange sex for drugs and other CSW

Rationale: these recommendations dovetail with USPSTF recommendations [28, 29] except with regard to adults in correctional facilities. According to Israeli correctional facility health reports (Aurkin-Tischler D, Israeli Prison Services. Personal communication, 2012), screening is not required because syphilis does not pose a serious threat to this population.

\section{Discussion}

Conservative $\mathrm{MoH}$ policy regarding STI screening in Israel reflects the relatively low national incidence of
STIs, although underestimation is almost certainly an important issue, similar to many countries [36, 37]. Underestimation results from passive surveillance systems' inability to capture true population STI incidence, most likely originating from structural and functional limitations, and threatens accurate reporting due to the intrinsic biases of these systems. Therefore we recommend implementing measures to optimize the existing passive surveillance system including supplementing it with a robust, active surveillance system (as has already been accomplished for HIV in Israel [38]).

\section{Developmental data objectives and capacity building}

a) Active surveillance Active surveillance projects should be undertaken in high risk population groups such CSW, IVDU (intravenous drug users), MSM, and HIV carriers, to rigorously establish the burden of disease in each subpopulation and be performed in sentinel locations such as STIs clinics, dedicated MSM and CSW venues.

b) Passive surveillance

The Israeli passive surveillance system should be revamped by utilizing the following methods:

A. Increased capacity building Additional professional personnel should be recruited by the MoH TB and AIDS department to enable it to also handle non-HIV STIs. This will facilitate improvements in STI surveillance and data processing, as well as other important tasks (e.g., development of field interventions and evaluation of such programs). Combined HIV and other STI surveillance, along with other activities has been done by several EU countries, such as France and the Netherlands (Caroline Semaille, from the "Institut de Veille Sanitaire" of France and Eline Op de Coul, from the Centre for Infectious Disease Control of The Netherland, April 2012, Personal communications).

B. Explore the adequacy of STI clinic health service coverage and staff data collection roles To determine the need for possible modification and improvements to existing services, population coverage of existing STI clinics, access thereof, and role definition of the staff may be further examined.

C. Improve the accuracy of clinical diagnoses

- Regularly update professional guidelines to reflect the state of the science;

- Establish a uniform, computerized case investigation form for use by epidemiologic nurses; 
- Provide a specific training course for STI clinic healthcare personnel: physicians, nurses, social workers, and train them to effectively interview patients and to improve epidemiological investigations;

- Increase the awareness of community-based primary care physicians and hospital staff of the need to culture patients with venereal secretions prior to initiating empiric treatment regimens;

- Ensure that PCR diagnostic tests are available in all community clinics to diagnose chlamydia and gonorrhea;

- Provide health providers with suitable equipment for sample collection, and increase their awareness of the proper methodology for sample transport and delivery;

- Improve laboratory assessment of antibiotic resistance in positive cultures, and assure timely notification of providers about specific diagnoses (as well as about the latest trends in microbial resistance in order to facilitate effective treatment);

- Authorize requests for Nucleic Acid Amplifications Test (NAAT) testing by primary care physicians nationwide, rather than requiring case-by-case approval by urologists;

- Enhance the diagnostic capability of the National STI's Laboratories (per disease);

- Consider data analysis using tools such as GIS [2].

\section{Developmental interventional objectives}

a) Behavioral surveillance and research should be expanded to better describe population risk behaviors and attitudes towards safe-sex to develop more effective interventions. Research indicates a relatively low awareness of STIs among primary health care clinicians [39]. Barriers and facilitators of clinician adherence to guidelines should be explored. The effectiveness of counseling should be assessed.

b) Improve contact tracing: Target populations of $\mathrm{TB}$ and STI programs are often similar and both utilize similar public health strategies. Rothenberg and others [40] have suggested expanding the contact tracing approach used by the national TB programs to STI programs (i.e., implementing horizontal integration of these programs). Innovative strategies such as identification of social networks should be adopted [2] that can be especially timely during periods of financial constraints [40]. Israel does not (yet) have a national STI program, but a national TB program has existed since 1997 [41]. However, implementing this scheme will require extensive planning (including development of appropriate training) to adapt these techniques to the specific needs of community health work to develop a focused contact tracing program [2].

c) A sexual health initiative may be progressively developed and implemented jointly by the Ministries of Health, Education, and Welfare. A recent example is Australia's Second National STI Strategy 2010-2013 [42].

d) Involve and empower additional health providers who treat potential patients. These include urologists, gynecologists, dermatologists, family physicians, and emergency room medical staff. Special effort should be invested to involve and continually update independent practitioners unaffiliated with the major HMOs.

e) The current AIDS national steering committee should be expanded to include several new members specializing in STI.

f) We also recommend performing cost-effectiveness analyses related to different components of future interventions as the second phase of this program.

\section{Conclusions}

A national STI (chlamydia, gonorrhea, and syphilis) prevention strategy for the year 2025 is presented. Although the current burden of STIs is relatively low compared to other Western countries, this is thought to be a partial result of underreporting. These and other gaps suggest a need for focused epidemiologic and health services research utilizing strategies such as active surveillance and field surveys to better characterize health risk behaviors as well as provider practice patterns. Primary preventive counseling is recommended for adolescents and younger adults. Due to the low prevalence of chlamydia and gonorrhea, only case finding in high risk populations is recommended. Screening with the VDRL test or the ELISA test is suggested for pregnant women, MSM, those exchanging sex for drugs, and CSW. Innovative implementation strategies include: cross-training of existing $\mathrm{MoH} \mathrm{TB}$ personnel, enhanced training and interface with community clinical specialists, and procurement of more advanced diagnostic and analytic tools at all levels of care, in conjunction with expanded authorization of healthcare personnel to avail themselves of these tools.

Implementation of these recommendations will require a commitment to reasonably increase the capacity of the Department of TB and AIDS and satellite organizations including increased staffing, development of 
specific training modules for infectious disease and primary care professionals, and major improvements of the existing technical infrastructure, including improvement in efficient and overall quality of laboratory diagnostic capabilities.

This should provide a strong foundation upon which to develop specific and implementable national STI prevention policy recommendations by 2025 .

\section{Abbreviations}

Chlamydia: Chlamydia trachomatis; CPSTF: Community Preventive Services Task Force; CRR: Comprehensive risk reduction; CSW: Commercial sex work; ECDC: European Centre for Disease Prevention and Control; ELISA: Enzyme-linked immunosorbent assay; EU: European Union; Gonorrhea: Neisseria gonorrhea; GUM clinics: Genitourinary medicine clinics; HIV: Human Immunodeficiency Virus; HMO: Health Maintenance Organization; IVDU: Intravenous drug users; MoH: Ministry of Health; MSM: Men having sex with men; NAAT: Nucleic Acid Amplifications Test; PCR: Polymerase chain reaction; PID: Pelvic inflammatory disease; STDs: Sexually transmitted diseases; STls: Sexually transmitted infections; Syphilis: Treponema pallidum; TB: Tuberculosis; UK: United Kingdom; US: United States of America; USPSTF: US Preventive Services Task Force; VDRL test: Venereal Disease Research Laboratory test; WHO: World Health Organization

\section{Acknowledgements}

We would like to acknowledged the contribution of the 'Healthy Israel 2020' "Tuberculosis, HIV and STI's" subcommittee members for their important contribution in the situation analysis and in the preliminary phase of setting priorities: Chemtob D (Chair), Chowers M, Dan M, Gandacu D, Shitrit D, Yust I. Thanks also to Dr. Emilia Anis, Chair of the Healthy Israel 2020 committee on "Infectious Diseases".

We would also like to thank the teams of the Division of Epidemiology (and specially Mr. Ruslan Gosinov) and of the District Health Offices for collecting and managing the data analyzed in this article, and all those who have notified these cases. Special thanks also to Ms. Nechama Averick for editorial support.

\section{Funding}

No source of funding was used.

\section{Availability of data and materials}

In order to have access to the data, please contact directly the corresponding author: daniel.chemtob@moh.gov.l.

\section{Authors' contributions}

DC initiated the article, prepared its design, led the overall analysis and international comparisons and drafted the manuscript, with a significant contribution from ER. DG and ZM participated in drafting the manuscript. DG and EA provided the Israeli STIs data. IG and EA also participated in the discussion and decisions processes related to these topics. All authors read and approved the final manuscript.

\section{Authors' information}

Dr. Daniel Chemtob MD, MPH, DEA, is a senior Public Health physician (MPH and DEA), with additional expertise in anthropology (DEA). Dr. Chemtob established the Department of Tuberculosis (TB) and AIDS at the Ministry of Health (in 1996) and the National TB program (in 1997) and continues to manage them. Dr. Chemtob has over 23 years of international experience in the fields of TB and HIV/AIDS, including 2 years as a TB Medical Officer at WHO-HQ. Dr. Chemtob has 1 year of graduate education and spent 2 years in the Post-Doctoral Fellowship at the Department of Epidemiology at the John Hopkins Bloomberg School of Public Health, Baltimore, USA and partly focused his activities on STIs prevention and treatment. Dr. Chemtob is a Senior Lecturer at the School of Public Health of Hebrew University of Jerusalem, Israel. daniel.chemtob@moh.health.gov.il.

Dr. Dan Gandacu MD, MPH is a senior Public Health practitioner and was in charge of the STI prevention and treatment in the past two decades until his retirement at the Division of Epidemiology, Ministry of Health, Jerusalem, Israel. dan.gandacu@moh.health.gov.il.
Dr. Zohar Mor MD, MPH, MHA, is a senior Public Health practitioner. He is currently a researcher at the Tel Aviv District Health Office and a senior lecturer at Tel Aviv University, Israel. zohar.mor@telaviv.health.gov.ll. Prof. Itamar Grotto MD, MPH, PhD, is a senior Public Health practitioner and the Director of the Israeli Public Health Services at the Ministry of Health for the past 9 years. He is a professor of Medicine and Epidemiology at the Department of Public Health at Ben-Gurion University, Beer Sheva, Israel. itamar.grotto@moh.health.gov.il.

Dr. Emilia Anis MD, MPH, is a senior Public Health practitioner and the Director of the Division of Epidemiology at the Ministry of Health. She is a senior lecturer at the Hebrew University of Jerusalem, Israel.

emilia.anis@moh.health.gov.l.

Dr. Elliot Rosenberg MD, MPH, is a senior Public Health and General Preventative Medicine specialist. He serves as the head of the Department of Occupational Health at the Ministry of Health. He also serves as the National Coordinator of the 'Healthy Israel 2020' initiative, tasked with creating a national preventative health blueprint for Israel over the decade spanning 2010-2020. In addition, he is a long-standing member of the Israeli Task Force for Health Promotion and Disease Prevention.

eli.rosenberg@moh.health.gov.il.

The opinions expressed in this article are those of the authors and do not purport to represent the opinions of the agencies with which they are associated.

\section{Competing interests}

The authors declare that they have no competing interests.

\section{Consent for publication}

Not applicable.

\section{Ethics approval and consent to participate}

Israeli data analyzed in this article were data obtained for surveillance purposes and therefore the need for approval was waived.

\section{Publisher's Note}

Springer Nature remains neutral with regard to jurisdictional claims in published maps and institutional affiliations.

\section{Author details \\ ${ }^{1}$ Department of Tuberculosis and AIDS, Ministry of Health, Jerusalem, Israel. ${ }^{2}$ Division of Epidemiology, Ministry of Health, Jerusalem, Israel. ${ }^{3}$ Tel Aviv Regional District Health Office, Ministry of Health \& School of Public Health, Tel Aviv University, Tel Aviv, Israel. ${ }^{4}$ Public Health Services, Ministry of Health, Jerusalem, Israel. ${ }^{5}$ Department of Occupational Health, Ministry of Health, Jerusalem, Israel.}

Received: 4 June 2016 Accepted: 21 March 2017

Published online: 19 April 2017

\section{References}

1. World Health Organization Regional Office for Europe. Centralized Information System for Infectious Diseases (CSID). Sexually Transmitted Infections. http://data.euro.who.int/cisid/. Accessed 27 Sept 2016.

2. Chemtob D, Zenilman JM, Gandacu D. What do we need to learn for a more comprehensive evidence-based policy decision on Sexually Transmitted Infections prevention and treatment in Israel? Int J STD \& AIDS. 2012;23:e11-5.

3. Rosenberg E, Lev B, Bin-Nun G, McKee M, Rosen L. Healthy Israel 2020: a visionary national health targeting initiative. Public Health. 2008:122:1217-25.

4. Chemtob D, Rosenberg E. Healthy Israel 2020: Objectives, targets, and evidence-based strategies to prevent tuberculosis and HIV infection in Israel. Int Public Health J. 2010;2:289-97.

5. US Department of Health and Human Services, Centers for Disease Control and Prevention. Selected STDs by Age, Race/Ethnicity, and Gender, 1996-2014 request. http://wonder.cdc.gov/std-race-age.html. Accessed 27 Sept 2016.

6. Australian Government Department of Health.National Notifiable Diseases Surveillance System. http://www9.health.gov.au/cda/source/rpt_4_sel.cfm; Accessed 27 Sept 2016.

7. Dan M, Mor Z, Gottliev S, Sheinberg B, Shohat T. Trends in antimicrobial susceptibility of Neisseria gonorrhoeae in Israel, 2002 to 2007, with special reference to fluoroquinolone resistance. Sex Transm Dis. 2010;37:451-3. 
8. Brosh-Nissimov T, Mor Z, Avramovich E, Katchman E, Avigdor B, Mor O, Turner D. Syphilis outbreak among men who have sex with men in Tel Aviv, Israel, 2008-2010. IMAJ. 2012;14(23):152-6.

9. Mor Z, Shohat T, Goor Y, Dan M. Risk behavior and sexually transmitted diseases in gay and heterosexual men attending an STD clinic in Tel Aviv, Israel: A cross-sectional study. IMAJ. 2012;14(3):147-51.

10. LeFevre ML, on behalf of the U.S. Preventive Services Task Force. Behavioral Counseling Interventions to Prevent Sexually Transmitted Infections: Draft Recommendation Statement. AHRQ Publication No. 13-05180-EF-2. Ann Intern Med. 2014;161(12):894-901.

11. Chin HB, Sipe TA, Elder RW, Mercer SL, Chattopadhyay SK, et al. Community Preventive Services Task Force. The effectiveness of group-based comprehensive risk-reduction and abstinence education interventions to prevent or reduce the risk of adolescent pregnancy, Human Immunodeficiency Virus, and sexually transmitted infections: two systematic reviews for the Guide to Community Preventive Services. Am J Prev Med. 2012;42:272-94.

12. Robin L, Dittus P, Whitaker D, Crosby R, Ethier K, Mezoff J, Miller K, PappasDeluca K. Behavioral interventions to reduce incidence of HIV, STD, and pregnancy among adolescents: a decade in review. J Adolesc Health. 2004; 34:3-26.

13. World Health Organization. Sexually transmitted infections. http://www.who. int/mediacentre/factsheets/fs110/en/. Accessed 28 Aug 2010.

14. United States Department of Health \& Human Services. Guide to Community Preventive Services. Sexual Behavior: youth development behavioral interventions coordinated with community service to reduce sexual risk behaviors in adolescents. www.thecommunityguide.org/hiv/ youthdev-community.html. Last updated: October 2007.

15. Clinical Guidelines, Israeli Task Force for Health Promotion and Disease Prevention, 2013 Edition. Tabenkin H, Lahad A editors. Ramat Gan: Israeli Medical Association and Israeli Association of Family Physicians; 2013.

16. Scholes D, Stergachis A, Heidrich FE, et al. Prevention of pelvic inflammatory disease by screening for cervical chlamydial infection. N Engl J Med. 1996; 334:1362-6.

17. Ostergaard L, Andersen B, Møller JK, Olesen F. Home sampling versus conventional swab sampling for screening of Chlamydia trachomatis in women: a cluster-randomised 1-year follow-up study. Clin Infect Dis. 2000; 31:951-7.

18. Centers for Disease Control and Prevention. CDC Grand Rounds: Chlamydia prevention: challenges and strategies for reducing disease burden and sequelae. MMWR. 2011;60:370-3.

19. Low N, Bender N, Nartey L, Shang A, Stephenson JM. Effectiveness of chlamydia screening: systematic review. Int J Epidemiol. 2009;38:435-48.

20. Low N, Redmond S, Uusküla A, van Bergen J, Ward H, Andersen B, Götz H. Screening for genital chlamydia infection. Cochrane Database Syst Rev. 2016:9:CD010866. doi:10.1002/14651858.CD010866.pub.

21. LeFevre ML, on behalf of the U.S. Preventive Services Task Force. Screening for Chlamydia and Gonorrhea: U.S. Preventive Services Task Force Recommendation Statement. Ann Intern Med. 2014;161:902-10. doi:10.7326/ M14-1981.

22. Mattei PL, Beachkofsky TM, Gilson RT, Wisco OJ. Syphilis: a reemerging infection. Am Fam Physician. 2012;86:433-40.

23. Hawkes S, Matin N, Broutet N, Low N. Effectiveness of interventions to improve screening for syphilis in pregnancy: a systematic review and metaanalysis. Lancet Infect Dis. 2011;11:684-91.

24. Barham L, Lewis $D$, Latimer N. One to one interventions to reduce sexually transmitted infections and under the age of 18 conceptions: a systematic review of the economic evaluations. Sex Transm Infect. 2007;83:441-7.

25. Chesson HW. Cost effectiveness of one to one STI prevention interventions. Sex Transm Infect. 2007;83:423-4.

26. Hu D, Hook 3rd EW, Goldie SJ. Screening for Chlamydia trachomatis in women 15 to 29 years of age: a cost-effectiveness analysis. Ann Intern Med. 2004; 141:501-13.

27. Turner K, Adams E, Grant A, Macleod J, Bell G, Clarke J, et al. Costs and cost effectiveness of different strategies for chlamydia screening and partner notification: an economic and mathematical modeling study. BMJ. 2010;341: c7250. doi:10.1136/bmj.c7250.

28. Screening for Syphilis Infection, Topic Page. July 2004. U.S. Preventive Services Task Force. http://www.uspreventiveservicestaskforce.org/uspstf/ uspssyph.htm. Accessed 15 Dec 2016.
29. Screening for syphilis infection in pregnancy. U.S. Preventive Services Task Force reaffirmation recommendation statement. U.S. Preventive Services Task Force. Ann Intern Med. 2009;150:705-9.

30. European Centre for Disease Prevention and Control. ECDC Guidance: Chlamydia control in Europe, June 2009. 2009. Stockholm. http://ecdc. europa.eu/en/publications/Publications/0906_GUI_Chlamydia_Control_in_ Europe.pdf. Accessed 18 Feb 2010.

31. National Health Service. Choices Chlamydia. 2015. http://www. chlamydiascreening.nhs.uk/ys/faq.html. Accessed 15 Dec 2016.

32. MacDonald N, Wong T. Canadian guidelines on sexually transmitted infections. CMAJ. 2007;2007:176. doi:10.1503/cmaj.061616.

33. Hocking JS, Walker J, Regan D, Chen MY, Fairley CK. Chlamydia screening Australia should strive to achieve what others have not. MJA. 2008;188:106-8.

34. UK Health Protection Agency and British Association of Sexual Health \& HIV. Guidance for gonorrhoea testing in England and Wales. 2010. Available from http://webarchive.nationalarchives.gov.uk/20140714084352/http:/www. hpa.org.uk/webc/HPAwebFile/HPAweb_C/1267550166455. Accessed 15 Dec 2016

35. French P, et al. IUSTI: 2008 European guidelines on the management of syphilis. Int J STD \& AIDS. 2009;20:300-9.

36. Nicoll A, Hamers FF. Are trends in HIV, gonorrhoea, and syphilis worsening in Western Europe? BMJ. 2002;324:1324-7.

37. European Centre for Disease Prevention and Control. Sexually transmitted infections in Europe 2013. Stockholm: ECDC; 2015.

38. Chemtob D, Grossman Z. The epidemiology of adult and adolescent HIV infection in Israel, a country of immigration. Int J STD AIDS. 2004;15:691-6.

39. Jenkins WD, Rabins C, Bhattacharya D. Importance of physicians in Chlamydia trachomatis control. Prev Med. 2011;53:335-7.

40. Rothenberg RB, McElroy PD, Wilce MA, Muth SQ. Contact tracing: comparing the approaches for sexually transmitted diseases and tuberculosis. Int J Tuberc Lung Dis. 2003;7:S342-8.

41. Chemtob D, Leventhal A, Berlowitz Y, Weiler-Ravell D. The new National Tuberculosis Control Programme in Israel, a country of high immigration. Int J Tuberc Lung Dis. 2003;7:828-36.

42. Australian Government. Department of Health. Second National Sexually Transmitted Infections Strategy, 2010-2013. Commonwealth of Australia, 2010. http://www.health.gov.au/internet/main/publishing.nsf/Content/ohpnational-strategies-2010-sti/\$File/sti.pdf. Accessed 29 Sept 2013.

\section{Submit your next manuscript to BioMed Central and we will help you at every step:}

- We accept pre-submission inquiries

- Our selector tool helps you to find the most relevant journal

- We provide round the clock customer support

- Convenient online submission

- Thorough peer review

- Inclusion in PubMed and all major indexing services

- Maximum visibility for your research

Submit your manuscript at www.biomedcentral.com/submit 